\title{
SENSITIVITY ANALYSIS OF PARAMETRIC VECTOR SET-VALUED OPTIMIZATION PROBLEMS VIA CODERIVATIVES
}

\author{
X. W. Xue ${ }^{1}$, S. J. Li ${ }^{1, *}$, C. M. Liao ${ }^{1}$ and Jen-Chih $\mathrm{Yao}^{2}$
}

\begin{abstract}
In this paper, by virtue of coderivatives, we investigate sensitivity analysis of parametric vector set-valued optimization problems in Banach spaces. Several examples are provided to illustrate the main results.
\end{abstract}

\section{INTRODUCTION}

Sensitivity analysis is not only theoretically interesting, but also practically important in optimization theory and in theory of variational inequality. Under suitable assumptions, it consists in the study of derivatives of perturbation maps. Some interesting results have been obtained in sensitivity analysis in vector optimization problems. By using the contingent derivative, Tanino [27] obtained the first results in this field. Various sensitivity analysis results in this direction can be found in Shi [26], Kuk, Tanino, and Tanaka [9], and so on. Bednarczuk and Song [2] studied sensitivity of a family of parametric optimization problems with set-valued maps via generalized contingent epiderivatives for a set-valued map (also see [3]). Using the so-called proto-differentiability (see [25]), Lee and Huy [10] proved that, under suitable conditions, the efficient point set-valued map is proto-differentiable. Recently, some authors have investigated the sensitivity properties of vector variational inequalities by virtue of the set-valued gap functions, (see, e.g., [12, 13, 15]).

The generalized derivatives mentioned above for set-valued maps are generated by tangent cones to their graphs in primal spaces. Another derivative-like construction for set-valued maps has been introduced by Mordukhovich [17] called coderivatives, which are generated by normal cones to their graphs in dual spaces.

Received August 9, 2010.

2010 Mathematics Subject Classification: 49K40, 90C29, 90C31, 49J53.

Key words and phrases: Parametric set-valued optimization, Efficient point set-valued map, (Fréchet) normal cone, (Fréchet) coderivative, Sensitivity analysis.

${ }^{1}$ This research was partially supported by the National Natural Science Foundation of China (Grant No. 10871216) and Chongqing University Postgraduates Science and Innovation Fund (Project No. 201005B1A0010338).

${ }^{2}$ The work was partially supported by the Grant NSC 99-2221-E-110-038-MY3.

*Corresponding author. 
The readers are referred to [19] for details on several types of coderivatives and particularly the normal coderivative, mixed coderivative and the Fréchet coderivative. The generalized derivatives and coderivatives are generally independent since there are normal cones that are not dual to any tangent ones, for instance the Mordukhovich normal cone (see, e.g., [19]). So it is meaningful to discuss the optimization problems via coderivatives instead of generalized derivatives. There are numerous applications of coderivatives to derive necessary conditions and existence properties in various vector optimization problems, such as $[1,5,22,28]$. Coderivatives have been also applied to sensitivity analysis of scalar (single-objective) optimization problems. We refer the readers to $[6,11,14,20,21,23,29]$ for just a few of them.

Recently, by using the known results in [19], Huy, Mordukhovich and Yao [7] obtained the sensitivity properties of the parametric vector optimization problems via the Mordukhovich coderivatives with respect to the so-called generalized order optimality in the Asplund space setting. Chuong and Yao [4] gave the sensitivity analysis results in parametric vector optimization problems by virtue of the Fréchet coderivative in the Banach space setting. However, it seems that there are not correct in the formulas for computing the coderivatives of sum of a set-valued map and a cone, and efficient point set-valued maps, respectively.

In this paper, we make an effort to investigate the sensitivity properties of parametric vector set-valued optimization problems via the coderivatives in Banach spaces. We establish the formulas for computing the Fréchet coderivatives, normal coderivative and mixed coderivative of the efficient point set-valued map, respectively. As a special case, we obtain the corresponding results for vector optimization problems, which modify the incorrect results in [4] and [7]. We also give some examples to illustrate these results. At the end, we specify the coderivatives formulas for the above efficient point set-valued map in parametric vector optimization problems involving explicit constraints of operator constraints.

The rest of the paper is organized as follows. In Section 2, we recall the basic definitions and notations from vector optimization, set-valued analysis and variational analysis. In Section 3, we first compute the coderivatives of a sum of a set-valued map and a cone, and then derive formulas for computing the coderivatives of efficient point set-valued maps in Banach spaces. Finally, we discuss the coderivatives of efficient point set-valued map of general parametric vector optimization problems.

Throughout the paper we use standard notation, with special symbols introduced where they are defined. Unless otherwise stated, all spaces considered are Banach whose norms are always denoted by $\|\cdot\|$. For any space $X$ we consider its dual space $X^{*}$ equipped with the weak* topology $w^{*}$, where $\langle\cdot, \cdot\rangle$ means the canonical pairing. The closed ball with center $x$ and radius $\eta$ is denoted by $B_{\eta}(x)$. The symbol $A^{*}$ denotes the adjoint operator of a linear continuous operator $A$. Let $K \subset Y$ be a 
closed and convex cone. The uniformly positive polar to cone $K$ is defined by

$$
K_{u p}^{*}:=\left\{y^{*} \in Y^{*} \mid \exists \beta>0,\left\langle y^{*}, k\right\rangle \geq \beta\|k\|, \forall k \in K\right\} .
$$

If $F: X \rightrightarrows Y$ is a set-valued map, we denote by $\operatorname{dom} F=\{x \in X \mid F(x) \neq \emptyset\}$ and $\operatorname{gph} F=\{(x, y) \in X \times Y \mid y \in F(x)\}$, the domain and graph of $F$, respectively. The notation $\stackrel{*}{\rightarrow}$ stands for weak $^{*}$ convergence in a dual space, while $x_{n} \stackrel{S}{\rightarrow} x$ means that the sequence $x_{n}$ is contained in the subset $S$ and converges to $x$. For set-valued map $F: X \rightrightarrows X^{*}$ the expression

$$
\begin{aligned}
\limsup _{x \rightarrow \bar{x}} F(x)= & \left\{x^{*} \in X^{*} \mid \exists \text { sequences } x_{k} \rightarrow \bar{x}, x_{k}^{*} \stackrel{*}{\rightarrow} x^{*} \text { s.t. } x_{k}^{*} \in F\left(x_{k}\right)\right. \\
& \text { for all } k \in \mathbb{N}\},
\end{aligned}
$$

signifies the sequential Painlevé-Kuratowski upper (outer) limit with respect to the norm topology in $X$ and the weak* topology in $X^{*} ; \mathbb{N}=\{1,2, \ldots\}$. The origins of all real normed spaces are denoted by 0 .

\section{Basic Definitions and Preliminaries}

In this section, we introduce the basic concepts and constructions of variational analysis and generalized differentiation needed for formulations and justifications of the main results of the paper. Most of the concepts and properties can be found in the recent monograph [19].

Definition 2.1. $[8,16]$. Let $\Omega \subset X$ be a nonempty subset of a Banach space.

(i) Given $\bar{x} \in \Omega$ and $\varepsilon \geq 0$. The set of $\varepsilon$-normals to $\Omega$ at $\bar{x} \in \Omega$ is defined by

$$
\hat{N}_{\varepsilon}(\bar{x}, \Omega)=\left\{x^{*} \in X^{*} \mid \limsup _{x \stackrel{\Omega}{\rightarrow} \bar{x}} \frac{\left\langle x^{*}, x-\bar{x}\right\rangle}{\|x-\bar{x}\|} \leq \varepsilon\right\} .
$$

When $\varepsilon=0$, the set (1) is a cone that is called the Fréchet normal cone (or the prenormal cone) to $\Omega$ at $\bar{x}$ and is denoted by $\hat{N}(\bar{x}, \Omega)$. We put $\hat{N}_{\varepsilon}(\bar{x}, \Omega)=\emptyset$ for all $\varepsilon \geq 0$ if $\bar{x} \notin \Omega$.

(ii) The Mordukhovich normal cone (or basic normal cone) to $\Omega \subset X$ at $\bar{x}$ is defined through the Painleve-Kuratowski upper (outer) limit as

$$
N(\bar{x}, \Omega)=\underset{x_{k} \rightarrow \bar{x}, \varepsilon_{k} \rightarrow 0_{+}}{\operatorname{Limsup}} \hat{N}_{\varepsilon_{k}}\left(x_{k}, \Omega\right)
$$

Definition 2.2. [17, 18]. Considering a set-valued map $\Phi: X \rightrightarrows Y$ between Banach spaces. 
(i) The $\varepsilon$-coderivative $\hat{D}_{\varepsilon}^{*} \Phi(\bar{x}, \bar{y})$ at $(\bar{x}, \bar{y})$ is defined through the $\varepsilon$-normal set (1) to the graph as

$$
\hat{D}_{\varepsilon}^{*} \Phi(\bar{x}, \bar{y})\left(y^{*}\right)=\left\{x^{*} \in X^{*} \mid\left(x^{*},-y^{*}\right) \in \hat{N}_{\varepsilon}((\bar{x}, \bar{y}), \operatorname{gph} \Phi)\right\} .
$$

When $\varepsilon=0$, the positive homogeneous set-valued map of $y^{*}$ in (3) is called the Fréchet coderivative of $\Phi$ at $(\bar{x}, \bar{y})$ and denoted by $\hat{D}^{*} \Phi(\bar{x}, \bar{y})(\cdot)$.

(ii) The normal (Mordukhovich) coderivative of $\Phi$ at $(\bar{x}, \bar{y})$ is

$$
D_{N}^{*} \Phi(\bar{x}, \bar{y})\left(y^{*}\right)=\left\{x^{*} \in X^{*} \mid\left(x^{*},-y^{*}\right) \in N((\bar{x}, \bar{y}), \operatorname{gph} \Phi)\right\} .
$$

that is, $D_{N}^{*} \Phi(\bar{x}, \bar{y})\left(y^{*}\right)$ is the collection of all $x^{*}$ for which there are sequences $\varepsilon_{k} \rightarrow 0_{+},\left(x_{k}, y_{k}\right) \rightarrow(\bar{x}, \bar{y}),\left(x_{k}^{*}, y_{k}^{*}\right) \stackrel{*}{\rightarrow}\left(x^{*}, y^{*}\right)$ with $\left(x_{k}, y_{k}\right) \in \operatorname{gph} \Phi$ and $x_{k}^{*} \in \hat{D}_{\varepsilon_{k}}^{*} \Phi\left(x_{k}, y_{k}\right)\left(y_{k}^{*}\right)$.

(iii) The mixed coderivative $D_{M}^{*} \Phi(\bar{x}, \bar{y})$ of a set-valued map $\Phi: X \rightrightarrows Y$ at $(\bar{x}, \bar{y})$ is the set-valued map $D_{M}^{*} \Phi(\bar{x}, \bar{y}): Y^{*} \rightrightarrows X^{*}$ defined by

$$
D_{M}^{*} \Phi(\bar{x}, \bar{y})\left(y^{*}\right)=\underset{\left(x_{k}, y_{k}, y_{k}^{*}\right) \rightarrow\left(\bar{x}, \bar{y}, y^{*}\right), \varepsilon_{k} \rightarrow 0_{+}}{\operatorname{Limsup}} \hat{D}_{\varepsilon_{k}}^{*} \Phi\left(x_{k}, y_{k}\right)\left(y_{k}^{*}\right),
$$

i.e., $x^{*} \in D_{M}^{*} \Phi(\bar{x}, \bar{y})\left(y^{*}\right)$ if and only if there are sequences $\varepsilon_{k} \rightarrow 0_{+}$, $\left(x_{k}, y_{k}, y_{k}^{*}\right) \rightarrow\left(\bar{x}, \bar{y}, y^{*}\right), x_{k}^{*} \stackrel{*}{\rightarrow} x^{*}$ with $\left(x_{k}, y_{k}\right) \in \operatorname{gph} \Phi$ and $x_{k}^{*} \in \hat{D}_{\varepsilon_{k}}^{*}$ $\Phi\left(x_{k}, y_{k}\right)\left(y_{k}^{*}\right)$.

One can equivalently put $\varepsilon_{k}=0$ in (2) and (5) if $F$ has closed graph around $(\bar{x}, \bar{y})$ and if both $X$ and $Y$ are Asplund, see [19,24]. It follows from the definitions that $D_{M}^{*} \Phi(\bar{x}, \bar{y})\left(y^{*}\right) \subset D_{N}^{*} \Phi(\bar{x}, \bar{y})\left(y^{*}\right)$ when the equality obviously holds if $Y$ is finite-dimensional. We say that $\Omega$ is regular at $\bar{x} \in \Omega$ if $N(\bar{x}, \Omega)=\hat{N}(\bar{x}, \Omega)$ and $\Phi$ is N-regular (resp. M-regular) at $(\bar{x}, \bar{y})$ if and only if $D_{N}^{*} \Phi(\bar{x}, \bar{y})=\hat{D}^{*} \Phi(\bar{x}, \bar{y})$ (resp. $D_{M}^{*} \Phi(\bar{x}, \bar{y})=\hat{D}^{*} \Phi(\bar{x}, \bar{y})$ ) (see [18]). The following proposition gives a sufficient condition for the regularity of $\Phi$ and special representations of the coderivatives.

Proposition 2.1. [19]. Let $\Phi: X \rightarrow Y$ be Frechet differentiable at $\bar{x}$. Then,

$$
\hat{D}^{*} \Phi(\bar{x})\left(y^{*}\right)=\left\{(\nabla \Phi(\bar{x}))^{*} y^{*}\right\}, \quad \forall y^{*} \in Y^{*} .
$$

Moreover, if $\Phi$ is strictly differentiable at $\bar{x}$, i.e., $\Phi$ is single-valued around $\bar{x}$ and

$$
\lim _{x, x^{\prime} \rightarrow \bar{x}}\left[\Phi(x)-\Phi\left(x^{\prime}\right)-\nabla \Phi(\bar{x})\left(x-x^{\prime}\right)\right] /\left\|x-x^{\prime}\right\|=0,
$$

then $\Phi$ is $N$-regular at $\bar{x}$ and one has

$$
D_{N}^{*} \Phi(\bar{x})\left(y^{*}\right)=D_{M}^{*} \Phi(\bar{x})\left(y^{*}\right)=\left\{(\nabla \Phi(\bar{x}))^{*} y^{*}\right\}, \quad \forall y^{*} \in Y^{*} .
$$


Definition 2.3. Let $f: X \rightarrow Y$ be a single-valued map and $\bar{x} \in \operatorname{dom} f$. $f$ is said to be local upper Lipschitzian at $\bar{x}$ if there are numbers $\eta>0$ and $L>0$ such that

$$
\|f(x)-f(\bar{x})\| \leq L\|x-\bar{x}\|, \text { for all } x \in B_{\eta}(\bar{x}) \cap \operatorname{dom} f .
$$

We say that a set-valued map $F: X \rightrightarrows Y$ admits a local upper Lipschitzian selection at $(\bar{x}, \bar{y}) \in \operatorname{gph} F$ if there is a single-valued map $f: \operatorname{dom} F \rightarrow Y$ which is local upper Lipschitzian at $\bar{x}$ satisfying $f(\bar{x})=\bar{y}$ and $f(x) \in F(x)$ for all $x \in \operatorname{dom} F$ in a neighborhood of $\bar{x}$. We say that $F$ admits a local upper Lipschitzian selection around $(\bar{x}, \bar{y}) \in \operatorname{gph} F$ if there is a neighborhood $U$ of $(\bar{x}, \bar{y})$ such that $F$ admits a local upper Lipschitzian selection at any $(x, y) \in \operatorname{gph} F \cap U$.

Now we recall some definitions and properties of Fréchet subdifferential of extended real-valued function. In [20], one can find proofs as well as related results and discussions. For an extended real-valued function $\phi: X \rightarrow \overline{\mathbb{R}}:=[-\infty, \infty]$, let epi $\phi=\{(x, \mu) \in X \times \mathbb{R} \mid \mu \geq \phi(x)\}$. The Fréchet lower subdifferential and Fréchet upper subdifferential of $\phi$ at $\bar{x}$ with $|\phi(\bar{x})|<\infty$ are defined, respectively, by

$$
\hat{\partial} \phi(\bar{x}):=\left\{x^{*} \in X^{*} \mid\left(x^{*},-1\right) \in \hat{N}((\bar{x}, \phi(\bar{x})), \text { epi } \phi)\right\},
$$

and

$$
\hat{\partial}^{+} \phi(\bar{x}):=-\hat{\partial}(-\phi)(\bar{x}) \text {. }
$$

It is known that the sets $\hat{\partial}^{+} \phi(\bar{x})$ and $\hat{\partial} \phi(\bar{x})$ are nonempty simultaneously if and only if $\phi$ is Fréchet differentiable at $\bar{x}$ with $\hat{\partial}^{+} \phi(\bar{x})=\hat{\partial} \phi(\bar{x})=\{\nabla \phi(\bar{x})\}$. Furthermore, for any vector function $f: P \rightarrow Y$ between Banach spaces we associate $f$ with a scalarization function with respect to some $y^{*} \in Y^{*}$ defined by

$$
\left\langle y^{*}, f\right\rangle(p)=\left\langle y^{*}, f(p)\right\rangle \text { for all } p \in P .
$$

If $f$ is locally upper Lipschitz continuous around $\bar{p} \in P$, then for all $y^{*} \in Y^{*}$,

$$
\hat{D}^{*} f(\bar{p})\left(y^{*}\right)=\hat{\partial}\left\langle y^{*}, f\right\rangle(\bar{p}) .
$$

\section{Main Results}

Let $F: P \times X \rightrightarrows Y$ and $C: P \rightrightarrows X$ be two set-valued maps, where $P, X$ and $Y$ are Banach spaces. Given a pointed (i.e., $K \cap(-K)=\{0\}$ ) closed convex cone $K \subset Y$, we consider the following parametric vector set-valued optimization problem:

$$
\operatorname{Min}_{K}\{F(p, x) \mid x \in C(p)\} .
$$

Here, $x$ is a decision variable and the cone $K$ includes a partial order $\preceq_{K}$ on $Y$, i.e.,

$$
y \preceq_{K} y^{\prime} \Leftrightarrow y^{\prime}-y \in K, \quad y, y^{\prime} \in Y .
$$


The " $M i n_{K}$ " in (6) is understood with respect to the ordering relation $\preceq_{K}$ from (7).

We say that $y \in A$ is an efficient point of a subset $A \in Y$ with respect to $K$ and write $y \in \operatorname{Min}_{K} A$, if and only if $(y-K) \cap A=\{y\}$. If $A=\emptyset$, then we define $\operatorname{Min}_{K} A=\emptyset$. Let $\bar{F}: P \rightrightarrows Y$ be the set-valued map given by

$$
\bar{F}(p)=(F \circ C)(p)=F(p, C(p))=\{F(p, x) \mid x \in C(p)\} .
$$

We put

$$
\mathcal{F}(p)=\operatorname{Min}_{K} \bar{F}(p), \quad p \in P,
$$

and call $\mathcal{F}: P \rightrightarrows Y$ the efficient point set-valued map of (6).

In this section, we derive sensitivity analysis of the parametric vector set-valued optimization problem (6). First, we need to compute or estimate the coderivatives of the sum of a set-valued map and a cone.

Proposition 3.1. Let $F: X \rightrightarrows Y$ be a set-valued map between Banach spaces and $(\bar{x}, \bar{y}) \in \operatorname{gph} F$. One has

$$
\hat{D}^{*}(F+K)(\bar{x}, \bar{y})\left(y^{*}\right) \subset \hat{D}^{*} F(\bar{x}, \bar{y})\left(y^{*}\right) \forall y^{*} \in Y^{*},
$$

and the converse inclusion holds if $y^{*} \in K_{u p}^{*}$ and $R$ admits a local upper Lipschitzian selection at $(\bar{x}, \bar{y}, \bar{y})$, where the set-valued map $R$ is defined by $R(x, y):=$ $F(x) \cap(y-K)$.

Proof. Clearly, $\operatorname{gph} F \subset \operatorname{gph}(F+K)$ since $0 \in K$. By the monotonicity property of the Frechet normal cone, we have

$$
\hat{N}((\bar{x}, \bar{y}), \operatorname{gph}(F+K)) \subset \hat{N}((\bar{x}, \bar{y}), \operatorname{gph} F),
$$

and it follows from the definition of the Fréchet coderivative that (10) holds. Let $y^{*} \in K_{u p}^{*}$ and $x^{*} \in \hat{D}^{*} F(\bar{x}, \bar{y})\left(y^{*}\right)$. Conversely, assume that $x^{*} \notin \hat{D}^{*}(F+$ $K)(\bar{x}, \bar{y})\left(y^{*}\right)$. This means that there is a sequence $\left(x_{k}, y_{k}\right) \rightarrow(\bar{x}, \bar{y})$ with $y_{k} \in$ $F\left(x_{k}\right)+K$ such that

$$
\limsup _{k \rightarrow \infty} \frac{\left\langle\left(x^{*},-y^{*}\right),\left(x_{k}-\bar{x}, y_{k}-\bar{y}\right)\right\rangle}{\left\|\left(x_{k}-\bar{x}, y_{k}-\bar{y}\right)\right\|}>0 .
$$

Since $R$ admits a local upper Lipschitzian selection at $(\bar{x}, \bar{y}, \bar{y})$ and $\operatorname{dom} R=$ $\operatorname{gph}(F+K)$, there are a constant $L>0$ and a neighborhood $U \times V$ of $(\bar{x}, \bar{y})$ such that for all $(x, y) \in(U \times V) \cap \operatorname{gph}(F+K)$, we can find $y_{1} \in R(x, y)$ such that

$$
\left\|y_{1}-\bar{y}\right\| \leq L\|(x-\bar{x}, y-\bar{y})\|
$$


For the above $\left(x_{k}, y_{k}\right)$, there exists $k_{0} \in \mathbb{N}$ such that for all $k \geq k_{0},\left(x_{k}, y_{k}\right) \in$ $U \times V$. Thus, there is a sequence $y_{k}^{\prime} \in R\left(x_{k}, y_{k}\right) \subset F\left(x_{k}\right)$ such that for $k$ large enough $\left\|y_{k}^{\prime}-\bar{y}\right\| \leq L\left\|\left(x_{k}, y_{k}\right)-(\bar{x}, \bar{y})\right\| \rightarrow 0$. That is, there are $y_{k}^{\prime} \in F\left(x_{k}\right)$ and $\theta_{k} \in K$ satisfying $y_{k}=y_{k}^{\prime}+\theta_{k}$ and $y_{k}^{\prime} \rightarrow \bar{y}$. Furthermore, by the choice of $y^{*} \in K_{u p}^{*}$, there exists $\beta>0$ satisfying $\left\langle y^{*}, k\right\rangle \geq \beta\|k\| \geq 0, \forall k \in K$. Therefore, we have found a sequence $\left(x_{k}, y_{k}^{\prime}\right) \stackrel{\operatorname{gph} F}{\longrightarrow}(\bar{x}, \bar{y})$ such that $\left\|\left(x_{k}-\bar{x}, y_{k}^{\prime}-\bar{y}\right)\right\| \leq$ $(L+1)\left\|\left(x_{k}, y_{k}\right)-(\bar{x}, \bar{y})\right\|$ and

$$
\begin{aligned}
& \frac{\left\langle\left(x^{*},-y^{*}\right),\left(x_{k}-\bar{x}, y_{k}^{\prime}-\bar{y}\right)\right\rangle}{\left\|\left(x_{k}-\bar{x}, y_{k}^{\prime}-\bar{y}\right)\right\|} \\
= & \frac{\left\langle\left(x^{*},-y^{*}\right),\left(x_{k}-\bar{x}, y_{k}-\theta_{k}-\bar{y}\right)\right\rangle}{\left\|\left(x_{k}-\bar{x}, y_{k}^{\prime}-\bar{y}\right)\right\|} \\
= & \frac{\left\langle\left(x^{*},-y^{*}\right),\left(x_{k}-\bar{x}, y_{k}-\bar{y}\right)\right\rangle+\left\langle y^{*}, \theta_{k}\right\rangle}{\left\|\left(x_{k}-\bar{x}, y_{k}^{\prime}-\bar{y}\right)\right\|} \\
\geq & \frac{\left\langle\left(x^{*},-y^{*}\right),\left(x_{k}-\bar{x}, y_{k}-\bar{y}\right)\right\rangle}{(L+1)\left\|\left(x_{k}-\bar{x}, y_{k}-\bar{y}\right)\right\|}+0 .
\end{aligned}
$$

It follows from (11) that

$$
\limsup _{(x, y) \stackrel{\operatorname{gph} F}{\longrightarrow}(\bar{x}, \bar{y})} \frac{\left\langle\left(x^{*},-y^{*}\right),(x-\bar{x}, y-\bar{y})\right\rangle}{\|(x-\bar{x}, y-\bar{y})\|}>0,
$$

and thus $x^{*} \notin \hat{D}^{*} F(\bar{x}, \bar{y})\left(y^{*}\right)$ from which it follows that the converse inclusion of (10) holds. The proof is now completed.

Remark 3.1. For the Fréchet coderivative, since there is a monotonicity property of the Fréchet normal cone, the inclusion relation holds automatically, and in most cases the inclusion relation is strict. So, in order to obtain the converse inclusion, strong assumptions should be added. On the one hand, the independent variable $y^{*}$ should be restricted on $K_{u p}^{*}$, since the left side of (10) may be equal to empty set in most cases of $y^{*} \notin K_{u p}^{*}$ (especially in finite dimensional spaces). On the other hand, the local upper Lipschitzian selection property of $R$ is important, which ensures that for any sequence of points tending to a given point in $\operatorname{gph}(F+K)$, one can take a subsequence tending to the given point in $\operatorname{gph} F$.

We give an example to show that the converse inclusion of (10) may fail to hold if the assumption on the existence of the local upper Lipschitzian selection of $R$ is omitted.

Example 3.1. Let $X=Y=\mathbb{R}, K=\mathbb{R}_{+}$and let $F: \mathbb{R} \rightrightarrows \mathbb{R}$ be defined by $F(x)=\left\{\begin{array}{ll}\{0,1\}, & x \geq 0 \\ \emptyset, & \text { else. }\end{array}\right.$ Then, $\operatorname{gph}(F+K)=\mathbb{R}_{+} \times \mathbb{R}_{+}$. Consider $(\bar{x}, \bar{y})=$ 
$(0,1)$ and $y^{*}=1 \in K_{u p}^{*}$. By computing, we obtain

$$
D_{N}^{*}(F+K)(0,1)(1)=\hat{D}^{*}(F+K)(0,1)(1)=\emptyset,
$$

and

$$
D_{N}^{*} F(0,1)(1)=\hat{D}^{*} F(0,1)(1)=\mathbb{R}_{-} .
$$

This means that Proposition 3.1 in [4] and Lemma 3.2 in [7] are not correct. In fact, consider $\left(x_{k}, y_{k}\right)=\left(\frac{1}{k}, 1-\frac{1}{k}\right) \rightarrow(\bar{x}, \bar{y})$ for $k \in \mathbb{N}$ large enough. We have $R\left(x_{k}, y_{k}\right)=\{0\}$. There does not exist a constant $L>0$ such that $\|0-\bar{y}\| \leq$ $L\left\|\left(x_{k}, y_{k}\right)-(\bar{x}, \bar{y})\right\|$. Thus, $R$ does not admit a local upper Lipschitzian selection at $(\bar{x}, \bar{y}, \bar{y})$.

In the sequel, let $D^{*}$ stand either for the normal coderivative (4) or for the mixed coderivative (5). Since the proofs of normal coderivative and mixed coderivative are similar, we only show the case of normal coderivative in the following.

Proposition 3.2. Let $F: X \rightrightarrows Y$ be a set-valued map between Banach spaces, $(\bar{x}, \bar{y}) \in \operatorname{gph} F$ and set-valued map $R$ be defined by $R(x, y):=F(x) \cap(y-K)$. If $R$ admits a local upper Lipschitzian selection at $(\bar{x}, \bar{y}, \bar{y})$, then one has

$$
D^{*}(F+K)(\bar{x}, \bar{y})\left(y^{*}\right) \subset D^{*} F(\bar{x}, \bar{y})\left(y^{*}\right), \quad \forall y^{*} \in Y^{*},
$$

and the converse inclusion holds if $y^{*} \in K_{u p}^{*}$ and $R$ admits a local upper Lipschitzian selection around $(\bar{x}, \bar{y}, \bar{y})$.

Proof. Firstly, we justify inclusion (12). Let $y^{*} \in Y^{*}$ and $x^{*} \in D_{N}^{*}(F+$ $K)(\bar{x}, \bar{y})\left(y^{*}\right)$. By the definitions of normal coderivative and normal cone, there are sequences $\varepsilon_{k} \rightarrow 0_{+},\left(x_{k}^{*}, y_{k}^{*}\right) \stackrel{*}{\rightarrow}\left(x^{*}, y^{*}\right)$ and $\left(x_{k}, y_{k}\right) \rightarrow(\bar{x}, \bar{y})$ with $y_{k} \in$ $(F+K)\left(x_{k}\right)$ such that

$$
\limsup _{(x, y) \stackrel{\operatorname{gph}(F+K)}{\longrightarrow}\left(x_{k}, y_{k}\right)} \frac{\left\langle\left(x_{k}^{*},-y_{k}^{*}\right),\left(x-x_{k}, y-y_{k}\right)\right\rangle}{\left\|\left(x-x_{k}, y-y_{k}\right)\right\|} \leq \varepsilon_{k} .
$$

Since $R$ admits a local upper Lipschitzian selection at $(\bar{x}, \bar{y}, \bar{y})$ and $\operatorname{dom} R=$ $\operatorname{gph}(F+K)$, there are a constant $L>0$ and a sequence $y_{k}^{\prime} \in R\left(x_{k}, y_{k}\right)$ such that $\left\|y_{k}^{\prime}-\bar{y}\right\| \leq L\left\|\left(x_{k}, y_{k}\right)-(\bar{x}, \bar{y})\right\|$. That is, there exists $\theta_{k} \in K$ such that $y_{k}^{\prime} \in F\left(x_{k}\right)$ and $y_{k}^{\prime}+\theta_{k}=y_{k}$. Now, we show that $x_{k}^{*} \in \hat{D}_{\varepsilon_{k}}^{*} F\left(x_{k}, y_{k}^{\prime}\right)\left(y_{k}^{*}\right)$. If not, there is a sequence $(\tilde{x}, \tilde{y}) \rightarrow\left(x_{k}, y_{k}^{\prime}\right)$ with $\tilde{y} \in F(\tilde{x})$ such that

$$
\lim \sup \frac{\left\langle\left(x_{k}^{*},-y_{k}^{*}\right),\left(\tilde{x}-x_{k}, \tilde{y}-y_{k}^{\prime}\right)\right\rangle}{\left\|\left(\tilde{x}-x_{k}, \tilde{y}-y_{k}^{\prime}\right)\right\|}>\varepsilon_{k} .
$$


Let $\tilde{y}^{\prime}:=\tilde{y}+\theta_{k}$. Then we have $\tilde{y}^{\prime}-y_{k}=\tilde{y}-y_{k}^{\prime},\left(\tilde{x}, \tilde{y}^{\prime}\right) \stackrel{\operatorname{gph}(F+K)}{\longrightarrow}\left(x_{k}, y_{k}\right)$ and $\limsup \frac{\left\langle\left(x_{k}^{*},-y_{k}^{*}\right),\left(\tilde{x}-x_{k}, \tilde{y}^{\prime}-y_{k}\right)\right\rangle}{\left\|\left(\tilde{x}-x_{k}, \tilde{y}^{\prime}-y_{k}\right)\right\|}=\limsup \frac{\left\langle\left(x_{k}^{*},-y_{k}^{*}\right),\left(\tilde{x}-x_{k}, \tilde{y}-y_{k}^{\prime}\right)\right\rangle}{\left\|\left(\tilde{x}-x_{k}, \tilde{y}-y_{k}^{\prime}\right)\right\|}>\varepsilon_{k}$,

which contradicts with (13). Thus, we have $x_{k}^{*} \in \hat{D}_{\varepsilon_{k}}^{*} F\left(x_{k}, y_{k}^{\prime}\right)\left(y_{k}^{*}\right)$ and then $x^{*} \in D_{N}^{*} F(\bar{x}, \bar{y})\left(y^{*}\right)$.

The converse inclusion can be proved similarly to the Fréchet coderivative in the above proposition. This completes the proof.

The following example shows that if we simply assume that $R$ admits a local upper Lipschitzian selection at $(\bar{x}, \bar{y}, \bar{y})$, the converse inclusion may not hold.

Example 3.2. Suppose that $X=Y=\mathbb{R}$ and $K=\mathbb{R}_{+}$. Let $F: X \rightrightarrows Y$ be defined by

$$
F(x)= \begin{cases}{\left[\frac{1}{4} x, \frac{1}{2} x\right] \cup[x, 2 x]} & \text { if } x \geq 0 \\ \emptyset & \text { else. }\end{cases}
$$

Observe that

$$
(F+K)(x)= \begin{cases}{\left[\frac{1}{4} x, \infty\right)} & \text { if } x \geq 0 \\ \emptyset & \text { else. }\end{cases}
$$

Consider $\bar{x}=\bar{y}=0$ and $y^{*}=1 \in K_{u p}^{*}$. It is obvious that $R$ admits a local upper Lipschitzian selection at $(\bar{x}, \bar{y}, \bar{y})$, but it does not admit a local upper Lipschitzian selection around $(\bar{x}, \bar{y}, \bar{y})$. By computing, we obtain

$$
D^{*}(F+K)(0,0)(1)=\left(-\infty, \frac{1}{4}\right],
$$

and

$$
D^{*} F(0,0)(1)=\left(-\infty, \frac{1}{4}\right] \cup\{1\} .
$$

This means that the converse inclusion of (12) fails to hold.

Now, we consider the coderivatives of the set-valued map $\bar{F}$ defined in (8). Let the set-valued map $M: P \times Y \rightrightarrows X$ be defined by

$$
M(p, y)=\{x \in X \mid y \in F(p, x) \text { and } x \in C(p)\} .
$$

If we define set-valued maps $\tilde{F}$ and $\tilde{C}$ as

$$
\operatorname{gph} \tilde{F}=\{(x, p, y) \mid y \in F(p, x)\} \quad \text { and } \operatorname{gph} \tilde{C}=\{(p, y, x) \mid x \in C(p)\},
$$

respectively, then $\operatorname{gph} M=\operatorname{gph} \tilde{F}^{-1} \cap \operatorname{gph} \tilde{C}$.

Recall that a set-valued map $F: X \rightrightarrows Y$ is said to be inner semicontinuous at $(\bar{x}, \bar{y}) \in \operatorname{gph} F$ if for every sequence $x_{k} \rightarrow \bar{x}$ there is a sequence $y_{k} \in F\left(x_{k}\right)$ converging to $\bar{y}$ as $k \rightarrow \infty$. 
Proposition 3.3. Let $P, X$ and $Y$ be Banach spaces and let $\bar{p} \in P, \bar{y} \in$ $(F \circ C)(\bar{p})$ where $F: P \times X \rightrightarrows Y$ and $C: P \rightrightarrows Y$ are two set-valued maps.

(i) Suppose that for some $\bar{x} \in M(\bar{p}, \bar{y})$ one has

$$
\begin{aligned}
& \hat{D}^{*} M(\bar{p}, \bar{y}, \bar{x})(0) \\
\subset & \bigcup_{\left(p^{*}, x^{*}\right) \in P^{*} \times X^{*}}\left(p^{*}+\hat{D}^{*} C(\bar{p}, \bar{x})\left(x^{*}\right)\right) \times \hat{D}^{*} F^{-1}(\bar{y}, \bar{p}, \bar{x})\left(-p^{*},-x^{*}\right) .
\end{aligned}
$$

Then for any $y^{*} \in Y^{*}$,

$$
\hat{D}^{*}(F \circ C)(\bar{p}, \bar{y})\left(y^{*}\right) \subset \bigcup_{\left(p^{*}, x^{*}\right) \in \hat{D}^{*} F(\bar{p}, \bar{x}, \bar{y})\left(y^{*}\right)} p^{*}+\hat{D}^{*} C(\bar{p}, \bar{x})\left(x^{*}\right) .
$$

(ii) Suppose that $M$ is inner semicontinuous at $(\bar{p}, \bar{y}, \bar{x})$ and

$$
D^{*} M(\bar{p}, \bar{y}, \bar{x})(0)
$$

$$
\subset \bigcup_{\left(p^{*}, x^{*}\right) \in P^{*} \times X^{*}}\left(p^{*}+D^{*} C(\bar{p}, \bar{x})\left(x^{*}\right)\right) \times D^{*} F^{-1}(\bar{y}, \bar{p}, \bar{x})\left(-p^{*},-x^{*}\right) .
$$

Then

$$
D^{*}(F \circ C)(\bar{p}, \bar{y})\left(y^{*}\right) \subset \bigcup_{\left(p^{*}, x^{*}\right) \in D^{*} F(\bar{p}, \bar{x}, \bar{y})\left(y^{*}\right)} p^{*}+D^{*} C(\bar{p}, \bar{x})\left(x^{*}\right) .
$$

Proof.

(i) For any $\bar{y}^{*} \in Y^{*}$, let $\bar{p}^{*} \in \hat{D}^{*}(F \circ C)(\bar{p}, \bar{y})\left(\bar{y}^{*}\right)$. By the definition of Fréchet coderivative, we have $\left(\bar{p}^{*},-\bar{y}^{*}\right) \in \hat{N}((\bar{p}, \bar{y}), \operatorname{gph}(F \circ C))$ and it follows from the definition of Fréchet normal cone that

$$
\limsup _{(p, y) \stackrel{\operatorname{gph}(F \circ C)}{\longrightarrow}(\bar{p}, \bar{y})} \frac{\left\langle\left(\bar{p}^{*},-\bar{y}^{*}\right),(p-\bar{p}, y-\bar{y})\right\rangle}{\|(p-\bar{p}, y-\bar{y})\|} \leq 0 .
$$

Then we have

$$
\begin{aligned}
& \limsup _{(p, y, x) \stackrel{\operatorname{gph} M}{\longrightarrow}(\bar{p}, \bar{y}, \bar{x})} \frac{\left\langle\left(\bar{p}^{*},-\bar{y}^{*}, 0\right),(p-\bar{p}, y-\bar{y}, x-\bar{x})\right\rangle}{\|(p-\bar{p}, y-\bar{y}, x-\bar{x})\|} \\
\leq & \limsup _{(p, y)} \frac{\left\langle\left(\bar{p}^{*},-\bar{y}^{*}\right),(p-\bar{p}, y-\bar{y})\right\rangle}{\|(p-\bar{p}, y-\bar{y})\|} \\
\leq & 0,
\end{aligned}
$$

which implies that $\left(\bar{p}^{*},-\bar{y}^{*}, 0\right) \in \hat{N}((\bar{p}, \bar{y}, \bar{x}), \operatorname{gph} M)$ and thus $\left(\bar{p}^{*},-\bar{y}^{*}\right) \in$ $\hat{D}^{*} M(\bar{p}, \bar{y}, \bar{x})(0)$. Condition (14) ensures that there exist $p^{*} \in P^{*}$ and $x^{*} \in X^{*}$ such that $-\bar{y}^{*} \in \hat{D}^{*} F^{-1}(\bar{y}, \bar{p}, \bar{x})\left(-p^{*},-x^{*}\right)$ hence $\left(p^{*}, x^{*}\right) \in$ $\hat{D}^{*} F(\bar{p}, \bar{x}, \bar{y})\left(\bar{y}^{*}\right)$, and $\bar{p}^{*} \in p^{*}+\hat{D}^{*} C(\bar{p}, \bar{x})\left(x^{*}\right)$. 
(ii) Let $\bar{p}^{*} \in D_{N}^{*}(F \circ C)(\bar{p}, \bar{y})\left(\bar{y}^{*}\right)$. By the definitions of normal coderivative and normal cone, there are sequences $\varepsilon_{k} \rightarrow 0_{+},\left(p_{k}, y_{k}\right) \rightarrow(\bar{p}, \bar{y})$ and $\left(p_{k}^{*}, y_{k}^{*}\right) \stackrel{*}{\rightarrow}\left(\bar{p}^{*}, \bar{y}^{*}\right)$ satisfying $y_{k} \in(F \circ C)\left(p_{k}\right)$ such that $p_{k}^{*} \in \hat{D}_{\varepsilon_{k}}^{*}(F \circ$ $C)\left(p_{k}, y_{k}\right)\left(y_{k}^{*}\right)$. Since $M$ is inner semicontinuous at $(\bar{p}, \bar{y}, \bar{x})$, for the above sequence $\left(p_{k}, y_{k}\right)$, there exists $x_{k} \in M\left(p_{k}, y_{k}\right)$ such that $x_{k} \rightarrow \bar{x}$. By similar proof as in (i), we have $\left(p_{k}^{*},-y_{k}^{*}\right) \in \hat{D}_{\varepsilon_{k}}^{*} M\left(p_{k}, y_{k}, x_{k}\right)(0)$ and hence $\left(\bar{p}^{*},-\bar{y}^{*}\right) \in D_{N}^{*} M(\bar{p}, \bar{y}, \bar{x})(0)$. Again by similar argument as in (i), (16) ensures (17). This completes the proof.

Remark 3.2. We remark that (14) and (16) are abstract assumptions. They can be replaced by

$$
\hat{N}((\bar{p}, \bar{y}, \bar{x}), \operatorname{gph} M) \subset \hat{N}((\bar{p}, \bar{y}, \bar{x}), \operatorname{gph} \tilde{C})+\hat{N}\left((\bar{p}, \bar{y}, \bar{x}), \operatorname{gph} \tilde{F}^{-1}\right),
$$

and

$$
N((\bar{p}, \bar{y}, \bar{x}), \operatorname{gph} M) \subset N((\bar{p}, \bar{y}, \bar{x}), \operatorname{gph} \tilde{C})+N\left((\bar{p}, \bar{y}, \bar{x}), \operatorname{gph} \tilde{F}^{-1}\right),
$$

respectively. In fact, if $\left(\bar{p}^{*}, \bar{y}^{*}\right) \in \hat{D}^{*} M(\bar{p}, \bar{y}, \bar{x})(0)$, then $\left(\bar{p}^{*}, \bar{y}^{*}, 0\right) \in \hat{N}((\bar{p}, \bar{y}, \bar{x})$, $\operatorname{gph} M)$. (18) ensures that one can find $p^{*}, p_{1}^{*} \in P^{*}, x^{*} \in X^{*}$ such that $\bar{p}^{*}=p_{1}^{*}+p^{*}$,

$$
\left(p_{1}^{*}, 0,-x^{*}\right) \in \hat{N}((\bar{p}, \bar{y}, \bar{x}), \operatorname{gph} \tilde{C}) \text { and }\left(p^{*}, \bar{y}^{*}, x^{*}\right) \in \hat{N}\left((\bar{p}, \bar{y}, \bar{x}), \operatorname{gph} \tilde{F}^{-1}\right) .
$$

By the construction of $\tilde{C}$ and $\tilde{F}^{-1}$, we have $\bar{y}^{*} \in \hat{D}^{*} F^{-1}(\bar{y}, \bar{p}, \bar{x})\left(-p^{*},-x^{*}\right)$ and $p_{1}^{*} \in \hat{D}^{*} C(\bar{p}, \bar{x})\left(x^{*}\right)$, which imply that (14) holds. The case of normal coderivative is similar.

If $P, X, Y$ are Asplund spaces, $F$ and $C$ have closed graphs and either $F$ is PSNC at $(\bar{p}, \bar{x}, \bar{y})$ or $C^{-1}$ is PSNC at $(\bar{x}, \bar{p}),(19)$ is ensured by the condition

$$
\left(-D_{M}^{*} F(\bar{p}, \bar{x}, \bar{y})(0)\right) \cap\left\{(0, v): v \in D_{M}^{*} C^{-1}(\bar{x}, \bar{p})(0)\right\}=\{(0,0)\} .
$$

One can see [19] for more details.

When $F$ is a vector-valued map, we have the following proposition.

Proposition 3.4. Let $P, X$ and $Y$ be Banach spaces and let $\bar{p} \in P, \bar{y} \in$ $(F \circ C)(\bar{p})$ where $F: P \times X \rightarrow Y$ is a vector-valued map and $C: P \rightrightarrows X$ is a set-valued map with closed graph.

(i) For $y^{*} \in Y^{*}$, suppose that for some $\bar{x} \in M(\bar{p}, \bar{y}), F$ is locally upper Lipschitzian at $(\bar{p}, \bar{x})$ and $\hat{\partial}^{+}\left\langle y^{*}, F\right\rangle(\bar{p}, \bar{x}) \neq \emptyset$. Then, one has

$$
\hat{D}^{*}(F \circ C)(\bar{p}, \bar{y})\left(y^{*}\right) \subset \bigcap_{\left(p^{*}, x^{*}\right) \in \hat{\partial}^{+}\left\langle y^{*}, F\right\rangle(\bar{p}, \bar{x})} p^{*}+\hat{D}^{*} C(\bar{p}, \bar{x})\left(x^{*}\right) .
$$

(ii) Suppose that all spaces are Asplund, $F$ is locally Lipschitzian at $(\bar{p}, \bar{x})$ and that $M$ is inner semicontinuous at $(\bar{p}, \bar{y}, \bar{x})$. Then, for any $y^{*} \in Y^{*}$, 


$$
D^{*}(F \circ C)(\bar{p}, \bar{y})\left(y^{*}\right) \subset \bigcup_{\left(p^{*}, x^{*}\right) \in D^{*} F(\bar{p}, \bar{x})\left(y^{*}\right)} p^{*}+D^{*} C(\bar{p}, \bar{x})\left(x^{*}\right) .
$$

Proof. The first part can be found in Proposition 3.3 in [4]. Suppose that $F$ is locally Lipschitzian at $(\bar{p}, \bar{x})$. Then, it is obvious that $F$ is PSNC at $(\bar{p}, \bar{y}, \bar{x})$. Moreover, by the Theorem 1.44 of [19], we have $D_{M}^{*} F(\bar{p}, \bar{x}, \bar{y})(0)=\{(0,0)\}$ and then the result immediately follows from Proposition 3.3 and Remark 3.2.

Further, if $F$ is differentiable at the reference point, we have the following corollary.

Corollary 3.1. If $F$ is a single-valued map from $P \times X$ to $Y$ which is Frechet differentiable at $(\bar{p}, \bar{x})$ with the derivative $\nabla F(\bar{p}, \bar{x})=\left(\nabla_{p} F(\bar{p}, \bar{x}), \nabla_{x} F(\bar{p}, \bar{x})\right)$, then (14) holds and in this case,

$$
\hat{D}^{*}(F \circ C)(\bar{p}, \bar{y})\left(y^{*}\right) \subset \nabla_{p} F(\bar{p}, \bar{x})^{*} y^{*}+\hat{D}^{*} C(\bar{p}, \bar{x})\left(\nabla_{x} F(\bar{p}, \bar{x})^{*} y^{*}\right) .
$$

Moreover, if $F$ is strictly differentiable at $(\bar{p}, \bar{x})$ and $M$ is inner semicontinuous at $(\bar{p}, \bar{y}, \bar{x})$, then (16) holds and

$$
D^{*}(F \circ C)(\bar{p}, \bar{y})\left(y^{*}\right) \subset \nabla_{p} F(\bar{p}, \bar{x})^{*} y^{*}+D^{*} C(\bar{p}, \bar{x})\left(\nabla_{x} F(\bar{p}, \bar{x})^{*} y^{*}\right) .
$$

Proof. Assume that $F$ is single-valued and Fréchet differentiable at $(\bar{p}, \bar{x})$. For any $\left(\bar{p}^{*},-\bar{y}^{*}\right) \in \hat{D}^{*} M(\bar{p}, \bar{y}, \bar{x})(0)$, by the definitions of Fréchet coderivative and Fréchet normal cone, we have

$$
\limsup _{(p, y, x) \stackrel{\operatorname{gph} M}{\longrightarrow}(\bar{p}, \bar{y}, \bar{x})} \frac{\left\langle\left(\bar{p}^{*},-\bar{y}^{*}, 0\right),(p-\bar{p}, y-\bar{y}, x-\bar{x})\right\rangle}{\|(p-\bar{p}, y-\bar{y}, x-\bar{x})\|} \leq 0 .
$$

Since $F$ is Fréchet differentiable at $(\bar{p}, \bar{x})$, one has $\hat{D}^{*} F(\bar{p}, \bar{x}, \bar{y})\left(\bar{y}^{*}\right)=\left(\nabla_{p} F(\bar{p}, \bar{x})^{*} \bar{y}^{*}\right.$, $\left.\nabla_{x} F(\bar{p}, \bar{x})^{*} \bar{y}^{*}\right)$ which implies that $-\bar{y}^{*} \in \hat{D}^{*} F^{-1}(\bar{y}, \bar{p}, \bar{x})\left(-\nabla_{p} F(\bar{p}, \bar{x})^{*} \bar{y}^{*},-\nabla_{x}\right.$ $\left.F(\bar{p}, \bar{x})^{*} \bar{y}^{*}\right)$. To justify the inclusion relation (14), we only need to show $\bar{p}^{*}-$ $\nabla_{p} F(\bar{p}, \bar{x})^{*} \bar{y}^{*} \in \hat{D}^{*} C(\bar{p}, \bar{x})\left(\nabla_{x} F(\bar{p}, \bar{x})^{*} \bar{y}^{*}\right)$. In fact, we have $\|(p-\bar{p}, y-\bar{y}, x-$ $\bar{x})\|\leq(\|\nabla F(\bar{p}, \bar{x})\|+2)\|(p-\bar{p}, x-\bar{x}) \|$. Then,

$$
\begin{aligned}
& \limsup _{(p, x) \stackrel{\operatorname{gphC}}{\longrightarrow}(\bar{p}, \bar{x})} \frac{\left\langle\left(\bar{p}^{*}-\nabla_{p} F(\bar{p}, \bar{x})^{*} \bar{y}^{*},-\nabla_{x} F(\bar{p}, \bar{x})^{*} \bar{y}^{*}\right),(p-\bar{p}, x-\bar{x})\right\rangle}{\|(p-\bar{p}, x-\bar{x})\|} \\
= & \limsup _{(p, x)} \frac{\left\langle\left(\bar{p}^{*},-\bar{y}^{*}\right),(p-\bar{p}, \nabla F(\bar{p}, \bar{x})(p-\bar{p}, x-\bar{x}))\right\rangle}{\|(p-\bar{p}, \bar{x})} \\
\leq & t \quad \limsup _{(p, y, x) \stackrel{\operatorname{gph} M}{\longrightarrow}(\bar{p}, \bar{y}, \bar{x})} \frac{\left\langle\left(\bar{p}^{*},-\bar{y}^{*}, 0\right),(p-\bar{p}, y-\bar{y}, x-\bar{x})\right\rangle}{\|(p-\bar{p}, y-\bar{y}, x-\bar{x})\|}
\end{aligned}
$$




$$
\begin{aligned}
& +\limsup _{(p, x) \stackrel{\text { gphC }}{\longrightarrow}(\bar{p}, \bar{x})} \frac{\circ(\|(p-\bar{p}, x-\bar{x})\|)}{\|(p-\bar{p}, x-\bar{x})\|} \\
& \leq 0,
\end{aligned}
$$

where $t=\|\nabla F(\bar{p}, \bar{x})\|+2$. By the definition of Fréchet coderivative, we have $\bar{p}^{*}-\nabla_{p} F(\bar{p}, \bar{x})^{*} \bar{y}^{*} \in \hat{D}^{*} C(\bar{p}, \bar{x})\left(\nabla_{x} F(\bar{p}, \bar{x})^{*} \bar{y}^{*}\right)$ and then (14) holds.

If $F$ is strictly differentiable at $(\bar{p}, \bar{x})$, similar argument ensures (16) since $D_{N}^{*} F(\bar{p}, \bar{x}, \bar{y})\left(y^{*}\right)=D_{M}^{*} F(\bar{p}, \bar{x}, \bar{y})\left(y^{*}\right)=(\nabla F(\bar{p}, \bar{x}))^{*} y^{*}$.

Now, let us turn to the reverse inclusions of (15) and (17).

Proposition 3.5. Suppose that $M$ admits a local upper Lipschitzian selection at $(\bar{p}, \bar{y}, \bar{x})$. Then for any $y^{*} \in Y^{*}$,

$$
\bigcup_{\left(p^{*}, x^{*}\right) \in \hat{D}^{*} F(\bar{p}, \bar{x}, \bar{y})\left(y^{*}\right)} p^{*}+\hat{D}^{*} C(\bar{p}, \bar{x})\left(x^{*}\right) \subset \hat{D}^{*}(F \circ C)(\bar{p}, \bar{y})\left(y^{*}\right) .
$$

Moreover, if for any $\left(p^{*}, x^{*}\right) \in P^{*} \times X^{*}$, one has

$$
\left(p^{*}+D^{*} C(\bar{p}, \bar{x})\left(x^{*}\right)\right) \times D^{*} F^{-1}(\bar{y}, \bar{p}, \bar{x})\left(-p^{*},-x^{*}\right) \subset \hat{D}^{*} M(\bar{p}, \bar{y}, \bar{x})(0),
$$

(or $M$ admits a local upper Lipschitzian selection around $(\bar{p}, \bar{y}, \bar{x})$ and

$$
\left(p^{*}+D^{*} C(\bar{p}, \bar{x})\left(x^{*}\right)\right) \times D^{*} F^{-1}(\bar{y}, \bar{p}, \bar{x})\left(-p^{*},-x^{*}\right) \subset D_{M}^{*} M(\bar{p}, \bar{y}, \bar{x})(0)
$$

holds, ) then for any $y^{*} \in Y^{*}$,

$$
\bigcup_{\left(p^{*}, x^{*}\right) \in D^{*} F(\bar{p}, \bar{x}, \bar{y})\left(y^{*}\right)} p^{*}+D^{*} C(\bar{p}, \bar{x})\left(x^{*}\right) \subset D^{*}(F \circ C)(\bar{p}, \bar{y})\left(y^{*}\right) .
$$

Proof. Let $\left(p^{*}, x^{*}\right) \in \hat{D}^{*} F(\bar{p}, \bar{x}, \bar{y})\left(y^{*}\right)$ and $p_{1}^{*} \in \hat{D}^{*} C(\bar{p}, \bar{x})\left(x^{*}\right)$. By the definition of Fréchet coderivative, $\left(p^{*}, x^{*},-y^{*}\right) \in \hat{N}((\bar{p}, \bar{x}, \bar{y}), \operatorname{gph} F)$ and $\left(p_{1}^{*},-x^{*}\right) \in$ $\hat{N}((\bar{p}, \bar{x}), \operatorname{gph} C)$. By the definitions of $\tilde{F}$ and $\tilde{C}$, we have $\left(p^{*},-y^{*}, x^{*}\right) \in \hat{N}((\bar{p}, \bar{y}, \bar{x})$, $\left.\operatorname{gph} \tilde{F}^{-1}\right)$ and $\left(p_{1}^{*}, 0,-x^{*}\right) \in \hat{N}((\bar{p}, \bar{y}, \bar{x})$, gph $\tilde{C})$. The monotonicity property and convexity of the Fréchet normal cone ensure that

$$
\left(p_{1}^{*}+p^{*},-y^{*}, 0\right) \in \hat{N}\left((\bar{p}, \bar{y}, \bar{x}), \operatorname{gph} \tilde{F}^{-1}\right)+\hat{N}((\bar{p}, \bar{y}, \bar{x}), \operatorname{gph} \tilde{C}) \subset \hat{N}((\bar{p}, \bar{y}, \bar{x}), \operatorname{gph} M) .
$$

By the definition of Fréchet normal cone, we have

$$
\limsup _{(p, y, x) \stackrel{\operatorname{gph} M}{\longrightarrow}(\bar{p}, \bar{y}, \bar{x})} \frac{\left\langle\left(p_{1}^{*}+p^{*},-y^{*}, 0\right),(p-\bar{p}, y-\bar{y}, x-\bar{x})\right\rangle}{\|(p-\bar{p}, y-\bar{y}, x-\bar{x})\|} \leq 0 .
$$


Suppose that $p_{1}^{*}+p^{*} \notin \hat{D}^{*}(F \circ C)(\bar{p}, \bar{y})\left(y^{*}\right)$. Then, there is a sequence $(p, y) \rightarrow$ $(\bar{p}, \bar{y})$ with $y \in(F \circ C)(p)$ such that

$$
\limsup _{(p, y) \stackrel{\operatorname{gph}(F \circ C)}{\longrightarrow}(\bar{p}, \bar{y})} \frac{\left\langle\left(p_{1}^{*}+p^{*},-y^{*}\right),(p-\bar{p}, y-\bar{y})\right\rangle}{\|(p-\bar{p}, y-\bar{y})\|}>0 .
$$

Since $M$ admits a local upper Lipschitzian selection at $(\bar{p}, \bar{y}, \bar{x})$, for the above $(p, y)$, there exist $x \in M(p, y)$ and a constant $L>0$ such that $\|x-\bar{x}\| \leq L\|(p-\bar{p}, y-\bar{y})\|$. Thus,

$$
\|(p-\bar{p}, y-\bar{y}, x-\bar{x})\| \leq(L+1)\|(p-\bar{p}, y-\bar{y})\| .
$$

Therefore, we can find a sequence $(p, y, x) \rightarrow(\bar{p}, \bar{y}, \bar{x})$ satisfying $x \in M(p, y)$ such that

$$
\begin{aligned}
& \limsup _{(p, y, x) \stackrel{\operatorname{gph} M}{\longrightarrow}(\bar{p}, \bar{y}, \bar{x})} \frac{\left\langle\left(p_{1}^{*}+p^{*},-y^{*}, 0\right),(p-\bar{p}, y-\bar{y}, x-\bar{x})\right\rangle}{\|(p-\bar{p}, y-\bar{y}, x-\bar{x})\|} \\
& \geq \frac{1}{(L+1)} \limsup _{(p, y) \stackrel{\operatorname{gph}(F \circ C)}{\longrightarrow}(\bar{p}, \bar{y})} \frac{\left\langle\left(p_{1}^{*}+p^{*},-y^{*}\right),(p-\bar{p}, y-\bar{y})\right\rangle}{\|(p-\bar{p}, y-\bar{y})\|} \\
& >0 \text {, }
\end{aligned}
$$

which is a contradiction. So, (22) holds.

Now, assume that $\left(\bar{p}^{*}, \bar{x}^{*}\right) \in D^{*} F(\bar{p}, \bar{x}, \bar{y})\left(\bar{y}^{*}\right)$ and $\bar{p}_{1}^{*} \in D^{*} C(\bar{p}, \bar{x})\left(\bar{x}^{*}\right)$. Then we have $-\bar{y}^{*} \in D^{*} F^{-1}(\bar{y}, \bar{p}, \bar{x})\left(-\bar{p}^{*},-\bar{x}^{*}\right)$. (23) ensures that $\left(\bar{p}_{1}^{*}+\bar{p}^{*},-\bar{y}^{*}\right) \in$ $\hat{D}^{*} M(\bar{p}, \bar{y}, \bar{x})(0)$. It follows from the first part of the proof that $\bar{p}_{1}^{*}+\bar{p}^{*} \in \hat{D}^{*}(F \circ$ $C)(\bar{p}, \bar{y})\left(\bar{y}^{*}\right)$ and hence $\bar{p}_{1}^{*}+\bar{p}^{*} \in D^{*}(F \circ C)(\bar{p}, \bar{y})\left(\bar{y}^{*}\right)$.

For the case of $M$ admits a local upper Lipschitzian selection around $(\bar{p}, \bar{y}, \bar{x})$, similar proof shows that $\left(\bar{p}_{1}^{*}+\bar{p}^{*},-\bar{y}^{*}\right) \in D_{M}^{*} M(\bar{p}, \bar{y}, \bar{x})(0)$. Arguing by contradiction, suppose that $\bar{p}_{1}^{*}+\bar{p}^{*} \notin D_{N}^{*}(F \circ C)(\bar{p}, \bar{y})\left(\bar{y}^{*}\right)$. Then for any sequences $\varepsilon_{k} \rightarrow 0_{+}$, $\left(p_{k}, y_{k}\right) \rightarrow(\bar{p}, \bar{y})$ and $\left(p_{k}^{*}, y_{k}^{*}\right) \stackrel{*}{\rightarrow}\left(\bar{p}^{*}+\bar{p}_{1}^{*}, \bar{y}^{*}\right)$ satisfying $y_{k} \in(F \circ C)\left(x_{k}\right)$, we have

$$
\limsup _{(p, y) \stackrel{\operatorname{gph}(F \circ C)}{\longrightarrow}\left(p_{k}, y_{k}\right)} \frac{\left\langle\left(p_{k}^{*},-y_{k}^{*}\right),\left(p-p_{k}, y-y_{k}\right)\right\rangle}{\left\|\left(p-p_{k}, y-y_{k}\right)\right\|}>\varepsilon_{k} .
$$

Since $M$ admits a local upper Lipschitzian selection around $(\bar{p}, \bar{y}, \bar{x})$, for the above $(p, y),\left(p_{k}, y_{k}\right)$ and any $x_{k} \in M\left(p_{k}, y_{k}\right)$ with $x_{k} \rightarrow \bar{x}$, there exist $x \in M(p, y)$ and a constant $L>0$ such that $\left\|x-x_{k}\right\| \leq L\left\|\left(p-p_{k}, y-y_{k}\right)\right\|$. Thus,

$$
\left\|\left(p-p_{k}, y-y_{k}, x-x_{k}\right)\right\| \leq(L+1)\left\|\left(p-p_{k}, y-y_{k}\right)\right\| .
$$

Therefore, for any $x_{k}^{*} \rightarrow 0$ with $(L+1)\left\|x_{k}^{*}\right\|<\varepsilon_{k}$, 


$$
\begin{aligned}
& \limsup _{(p, y, x) \stackrel{\operatorname{gph} M}{\longrightarrow}\left(p_{k}, y_{k}, x_{k}\right)} \frac{\left\langle\left(p_{k}^{*},-y_{k}^{*}, x_{k}^{*}\right),\left(p-p_{k}, y-y_{k}, x-x_{k}\right)\right\rangle}{\left\|\left(p-p_{k}, y-y_{k}, x-x_{k}\right)\right\|}
\end{aligned}
$$

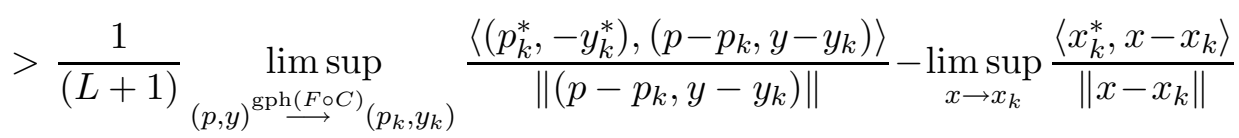

$$
\begin{aligned}
& >\frac{1}{(L+1)} \varepsilon_{k}-\left\|x_{k}^{*}\right\|:=\varepsilon_{k}^{\prime}>0 \text {, }
\end{aligned}
$$

which implies $\left(p_{k}^{*},-y_{k}^{*}\right) \notin \hat{D}_{\varepsilon_{k}^{\prime}}^{*} M\left(p_{k}, y_{k}, x_{k}\right)\left(-x_{k}^{*}\right)$ and therefore $\left(\bar{p}^{*}+\bar{p}_{1}^{*},-\bar{y}^{*}\right) \notin$ $D_{M}^{*} M(\bar{p}, \bar{y}, \bar{x})(0)$. This is a contradiction and then the proof is completed.

\section{Remark 3.3.}

(i) From the above proof, a sufficient condition under which (23) and (24) hold, is that $F$ and $C$ are N-regular at $(\bar{p}, \bar{x}, \bar{y})$ and $(\bar{p}, \bar{x})$, respectively. In fact, for mixed coderivative, we only need the M-regularity.

(ii) From the above proof, the assumption that $M$ has the local upper Lipschitzian selection property is essential. It ensures that the selected sequence not only connects with $C$ but also connects with $F$. If we simply assume that $C$ admits a local upper Lipschitzian selection at the reference point, the conclusion may not hold as the following example shows.

Example 3.3. Let $P=X=Y=\mathbb{R}, C(p)=\left\{\begin{array}{ll}{[0, \sqrt{p}],} & p \geq 0 \\ \emptyset, & \text { else, }\end{array}\right.$ and $F(p, x)=x^{2}$.

Then we have

$$
(F \circ C)(p)=\left\{\begin{array}{ll}
{[0, p],} & p \geq 0 \\
\emptyset, & \text { else, }
\end{array} \quad \text { and } M(p, y)= \begin{cases}\{0\}, & p \geq 0, y=0 \\
\sqrt{y}, & p \geq y>0 \\
\emptyset, & \text { else }\end{cases}\right.
$$

Consider $\bar{p}=\bar{x}=\bar{y}=0$. Obviously, $C$ admits a local upper Lipschitzian selection at $(\bar{p}, \bar{x})$, but $M$ does not admit a local upper Lipschitzian selection at $(\bar{p}, \bar{y}, \bar{x})$.

By direct computation, we obtain

$$
\begin{aligned}
\hat{D}^{*} C(\bar{p}, \bar{x})\left(x^{*}\right) & = \begin{cases}\mathbb{R}_{-}, & x^{*} \geq 0 \\
\emptyset, & \text { else },\end{cases} \\
\hat{D}^{*}(F \circ C)(\bar{p}, \bar{y})\left(y^{*}\right) & = \begin{cases}\mathbb{R}_{-}, & y^{*} \geq 0 \\
\left(-\infty, y^{*}\right] & \text { else, }\end{cases}
\end{aligned}
$$

and

$$
\hat{D}^{*} F(\bar{p}, \bar{x}, \bar{y})\left(y^{*}\right)=\{(0,0)\}, \quad \forall y^{*} \in \mathbb{R} .
$$

This means that the conclusion (22) does not hold. 
Corollary 3.2. If $F$ is a single-valued map from $P \times X$ to $Y$ which is Frechet differentiable at $(\bar{p}, \bar{x})$ with the derivative $\nabla F(\bar{p}, \bar{x})=\left(\nabla_{p} F(\bar{p}, \bar{x}), \nabla_{x} F(\bar{p}, \bar{x})\right)$ and $M$ admits a local upper Lipschitzian selection at $(\bar{p}, \bar{y}, \bar{x})$, then for any $y^{*} \in Y^{*}$,

$$
\nabla_{p} F(\bar{p}, \bar{x})^{*} y^{*}+\hat{D}^{*} C(\bar{p}, \bar{x})\left(\nabla_{x} F(\bar{p}, \bar{x})^{*} y^{*}\right) \subset \hat{D}^{*}(F \circ C)(\bar{p}, \bar{y})\left(y^{*}\right) .
$$

Moreover, if $F$ is strictly differentiable at $(\bar{p}, \bar{x})$ and $C$ is $N$-regular at $(\bar{p}, \bar{x})$, then for any $y^{*} \in Y^{*}$,

$$
\nabla_{p} F(\bar{p}, \bar{x})^{*} y^{*}+D^{*} C(\bar{p}, \bar{x})\left(\nabla_{x} F(\bar{p}, \bar{x})^{*} y^{*}\right) \subset D^{*}(F \circ C)(\bar{p}, \bar{y})\left(y^{*}\right) .
$$

Proof. It immediately follows from Proposition 3.5 and Remark 3.3.

Remark 3.4. Note that, in Example 3.3, $F$ is strictly differentiable at $(\bar{p}, \bar{x})$, $C$ is N-regular and admits a local upper Lipschitzian selection at $(\bar{p}, \bar{x})$. So, all the assumptions of Proposition 3.3 in [4] hold. However, (26) does not hold. So, Proposition 3.3 in [4] is not correct and the corresponding results in [4], which are related to Proposition 3.3, are also not correct.

We are now ready to formulate the main result of this section. Recall that a set-valued map $F: X \rightrightarrows Y$ is said to hold the domination property around $\bar{x}$ if there exists a neighborhood $U$ of $\bar{x}$ such that

$$
F(x) \subset \operatorname{Min}_{K} F(x)+K, \forall x \in U .
$$

Theorem 3.1. Let $\mathcal{F}$ be the efficient point set-valued map defined in (9) with order cone $K$ and let $\bar{p} \in P, \bar{y} \in \mathcal{F}(\bar{p})$. Suppose that the domination property holds for $\bar{F}$ around $\bar{p}$ and that $\mathcal{R}$ admits a local upper Lipschitzian selection around $(\bar{p}, \bar{y}, \bar{y})$, where the set-valued map $\mathcal{R}$ is defined by $\mathcal{R}(p, y):=\mathcal{F}(p) \cap(y-K)$.

(i) Suppose that for some $\bar{x} \in M(\bar{p}, \bar{y})$ one has

$$
\begin{aligned}
& \hat{D}^{*} M(\bar{p}, \bar{y}, \bar{x})(0) \\
\subset & \bigcup_{\left(p^{*}, x^{*}\right) \in P^{*} \times X^{*}}\left(p^{*}+\hat{D}^{*} C(\bar{p}, \bar{x})\left(x^{*}\right)\right) \times \hat{D}^{*} F^{-1}(\bar{y}, \bar{p}, \bar{x})\left(-p^{*},-x^{*}\right) .
\end{aligned}
$$

Then for any $y^{*} \in K_{u p}^{*}$,

$$
\hat{D}^{*} \mathcal{F}(\bar{p}, \bar{y})\left(y^{*}\right) \subset \bigcup_{\left(p^{*}, x^{*}\right) \in \hat{D}^{*} F(\bar{p}, \bar{x}, \bar{y})\left(y^{*}\right)} p^{*}+\hat{D}^{*} C(\bar{p}, \bar{x})\left(x^{*}\right) .
$$

(ii) Suppose that $M$ is inner semicontinuous at $(\bar{p}, \bar{y}, \bar{x})$ and 


$$
\begin{aligned}
& D^{*} M(\bar{p}, \bar{y}, \bar{x})(0) \\
\subset & \bigcup_{\left(p^{*}, x^{*}\right) \in P^{*} \times X^{*}}\left(p^{*}+D^{*} C(\bar{p}, \bar{x})\left(x^{*}\right)\right) \times D^{*} F^{-1}(\bar{y}, \bar{p}, \bar{x})\left(-p^{*},-x^{*}\right) .
\end{aligned}
$$

Then for any $y^{*} \in K_{u p}^{*}$,

$$
D^{*} \mathcal{F}(\bar{p}, \bar{y})\left(y^{*}\right) \subset \bigcup_{\left(p^{*}, x^{*}\right) \in D^{*} F(\bar{p}, \bar{x}, \bar{y})\left(y^{*}\right)} p^{*}+D^{*} C(\bar{p}, \bar{x})\left(x^{*}\right) .
$$

If $M$ admits a local upper Lipschitzian selection at $(\bar{p}, \bar{y}, \bar{x})$, then the converse inclusion of (28) holds. Furthermore, if for any $\left(p^{*}, x^{*}\right) \in P^{*} \times X^{*}$, one has

$$
\left(p^{*}+D^{*} C(\bar{p}, \bar{x})\left(x^{*}\right)\right) \times D^{*} F^{-1}(\bar{y}, \bar{p}, \bar{x})\left(-p^{*},-x^{*}\right) \subset \hat{D}^{*} M(\bar{p}, \bar{y}, \bar{x})(0),
$$

(or $M$ admits a local upper Lipschitzian selection around $(\bar{p}, \bar{y}, \bar{x})$ and

$$
\left(p^{*}+D^{*} C(\bar{p}, \bar{x})\left(x^{*}\right)\right) \times D^{*} F^{-1}(\bar{y}, \bar{p}, \bar{x})\left(-p^{*},-x^{*}\right) \subset D_{M}^{*} M(\bar{p}, \bar{y}, \bar{x})(0)
$$

holds, ) then the converse inclusion of (29) holds.

Proof.

(i) Since $\mathcal{F}(p) \subset \bar{F}(p)$ for all $p \in P$ and the domination property holds for $\bar{F}$ around $\bar{p}$, there is a neighborhood $U$ of $\bar{p}$ such that

$$
\mathcal{F}(p)+K=\bar{F}(p)+K, \quad \forall p \in U .
$$

Hence, for all $y^{*} \in Y^{*}$,

$$
\hat{D}^{*}(\mathcal{F}+K)(\bar{p}, \bar{y})\left(y^{*}\right)=\hat{D}^{*}(\bar{F}+K)(\bar{p}, \bar{y})\left(y^{*}\right) .
$$

Moreover, $\mathcal{F}(p) \subset \bar{F}(p)$ ensures that $\mathcal{R}(p, y) \subset R(p, y)$, where $R$ is defined by $R(p, y)=\bar{F}(p) \cap(y-K)$. Thus, $R(p, y)$ admits a local upper Lipschitzian selection at $(\bar{p}, \bar{y}, \bar{y})$ when $\mathcal{R}(p, y)$ admits a local upper Lipschitzian selection at $(\bar{p}, \bar{y}, \bar{y})$ and $\operatorname{gph}(\bar{F}+K)=\operatorname{gph}(\mathcal{F}+K)$. Applying the Proposition 3.1 twice, we get for all $y^{*} \in K_{u p}^{*}$,

$$
\hat{D}^{*}(\bar{F}+K)(\bar{p}, \bar{y})\left(y^{*}\right)=\hat{D}^{*} \bar{F}(\bar{p}, \bar{y})\left(y^{*}\right),
$$

and

$$
\hat{D}^{*}(\mathcal{F}+K)(\bar{p}, \bar{y})\left(y^{*}\right)=\hat{D}^{*} \mathcal{F}(\bar{p}, \bar{y})\left(y^{*}\right) .
$$

Therefore, we have for all $y^{*} \in K_{u p}^{*}$,

$$
\hat{D}^{*} \mathcal{F}(\bar{p}, \bar{y})\left(y^{*}\right)=\hat{D}^{*} \bar{F}(\bar{p}, \bar{y})\left(y^{*}\right) .
$$

Then Proposition 3.3 ensures (28) and Proposition 3.5 ensures the converse inclusion. 
(ii) By the definitions of $R$ and $\mathcal{R}$, if $\bar{F}$ holds the domination property around $\bar{p}$ and $\mathcal{R}$ admits a local upper Lipschitzian selection around $(\bar{p}, \bar{y}, \bar{y})$, then $R$ admits a local upper Lipschitzian selection around $(\bar{p}, \bar{y}, \bar{y})$. By similar argument as in (i), we can prove that the coderivatives formulas of $\mathcal{F}$ hold. This completes the proof.

If $F$ is a vector-valued map, then the parametric vector set-valued optimization problem (6) reduces to the conventional vector optimization problem.

Corollary 3.3. Let $F$ be a vector-valued map from $P \times X$ to $Y$. Suppose that the domination property holds for $\bar{F}$ around $\bar{p}$ and that $\mathcal{R}$ admits a local upper Lipschitzian selection around $(\bar{x}, \bar{y}, \bar{y})$.

(i) For $y^{*} \in K_{u p}^{*}$, suppose that for some $\bar{x} \in M(\bar{p}, \bar{y}), F$ is locally upper Lipschitzian at $(\bar{p}, \bar{x})$ and $\hat{\partial}^{+}\left\langle y^{*}, F\right\rangle(\bar{p}, \bar{x}) \neq \emptyset$. Then, one has

$$
\hat{D}^{*} \mathcal{F}(\bar{p}, \bar{y})\left(y^{*}\right) \subset \bigcap_{\left(p^{*}, x^{*}\right) \in \hat{\partial}^{+}\left\langle y^{*}, F\right\rangle(\bar{p}, \bar{x})} p^{*}+\hat{D}^{*} C(\bar{p}, \bar{x})\left(x^{*}\right) .
$$

(ii) Suppose that all spaces are Asplund, that $F$ is locally Lipschitzian at $(\bar{p}, \bar{x})$ and that $M$ is inner semicontinuous at $(\bar{p}, \bar{y}, \bar{x})$. Then, for any $y^{*} \in K_{u p}^{*}$,

$$
D^{*} \mathcal{F}(\bar{p}, \bar{y})\left(y^{*}\right) \subset \bigcup_{\left(p^{*}, x^{*}\right) \in D^{*} F(\bar{p}, \bar{x})\left(y^{*}\right)} p^{*}+D^{*} C(\bar{p}, \bar{x})\left(x^{*}\right) .
$$

(iii) If $F$ is Frechet differentiable at $(\bar{p}, \bar{x})$ with the derivative $\nabla F(\bar{p}, \bar{x})=$ $\left(\nabla_{p} F(\bar{p}, \bar{x}), \nabla_{x} F(\bar{p}, \bar{x})\right)$, then for any $y^{*} \in K_{u p}^{*}$,

$$
\hat{D}^{*} \mathcal{F}(\bar{p}, \bar{y})\left(y^{*}\right) \subset \nabla_{p} F(\bar{p}, \bar{x})^{*} y^{*}+\hat{D}^{*} C(\bar{p}, \bar{x})\left(\nabla_{x} F(\bar{p}, \bar{x})^{*} y^{*}\right) .
$$

(iv) If $F$ is strictly differentiable at $(\bar{p}, \bar{x})$ and $M$ is inner semicontinuous at $(\bar{p}, \bar{y}, \bar{x})$, then for any $y^{*} \in K_{u p}^{*}$,

$$
D^{*} \mathcal{F}(\bar{p}, \bar{y})\left(y^{*}\right) \subset \nabla_{p} F(\bar{p}, \bar{x})^{*} y^{*}+D^{*} C(\bar{p}, \bar{x})\left(\nabla_{x} F(\bar{p}, \bar{x})^{*} y^{*}\right) .
$$

If moreover, $M$ admits a local upper Lipschitzian selection at $(\bar{p}, \bar{y}, \bar{x})$, then the converse inclusion of (32) holds. Further, if $F$ is $N$-regular at $(\bar{p}, \bar{x}, \bar{y})$ and $C$ is $N$-regular at $(\bar{p}, \bar{x})$, then the converse inclusions of (31) and (33) hold.

Proof. It immediately follows from Proposition 3.1, Proposition 3.2, Proposition 3.4, Corollary 3.1 and Corollary 3.2.

We close this paper with an application to a special class of constrained vector optimization problem. More applications can be obtained similarly as in [4, 7]. 
Consider the parametric vector set-valued optimization problem (6) with the constraint set-valued map $C: P \rightrightarrows X$ given by

$$
C(p)=\{x \in X \mid h(p, x) \in \Theta\},
$$

where $h: P \times X \rightarrow Z$ is a single-valued mapping between Banach spaces, and where $\emptyset \neq \Theta \subset Z$. Constraints of type (34) are known as operator constraints.

The following theorem gives upper estimating and precise computing formulae to evaluate coderivatives of the optimization problem with constraints given by (34).

Theorem 3.2. Let $F$ be a single-valued map from $P \times X$ to $Y, \mathcal{F}$ be the efficient point set-valued map defined in (9) with the constraint mapping $C$ given by (34) and let $\bar{p} \in P, \bar{y} \in \mathcal{F}(\bar{p})$. Let set-valued map $S$ be defined by $S(p, y)=$ $\{x \in X \mid y \in F(p, x)$ and $h(p, x) \in \Theta\}$. Suppose that the domination property holds for $\bar{F}$ around $\bar{p}$, that $\mathcal{R}$ admits a local upper Lipschitzian selection around $(\bar{p}, \bar{y}, \bar{y})$ and that $h$ is strictly differentiable at $(\bar{p}, \bar{x})$ with the surjective derivative operator $\nabla h(\bar{p}, \bar{x})$.

(i) For $y^{*} \in K_{u p}^{*}$, suppose that for some $\bar{x} \in S(\bar{p}, \bar{y}), F$ is locally upper Lipschitzian at $(\bar{p}, \bar{x})$ and $\hat{\partial}^{+}\left\langle y^{*}, F\right\rangle(\bar{p}, \bar{x}) \neq \emptyset$. Then, one has

$$
\subset \bigcap_{\left(p^{*}, x^{*}\right) \in \hat{\partial}^{+}\left\langle y^{*}, F\right\rangle(\bar{p}, \bar{x})}\left\{p^{*}+u^{*} \mid\left(u^{*},-x^{*}\right) \in \nabla h(\bar{p}, \bar{x})^{*} \hat{N}((\bar{p}, \bar{x}), \Theta)\right\} .
$$

(ii) Suppose that all spaces are Asplund, $F$ is locally Lipschitzian at $(\bar{p}, \bar{x})$ and that $S$ is inner semicontinuous at $(\bar{p}, \bar{y}, \bar{x})$. Then, for any $y^{*} \in K_{u p}^{*}$,

$$
D^{*} \mathcal{F}(\bar{p}, \bar{y})\left(y^{*}\right) \subset \bigcup_{\left(p^{*}, x^{*}\right) \in D^{*} F(\bar{p}, \bar{x})\left(y^{*}\right)} p^{*}+D^{*} C(\bar{p}, \bar{x})\left(x^{*}\right) .
$$

(iii) If $F$ is Frechet differentiable at $(\bar{p}, \bar{x})$ with the derivative $\nabla F(\bar{p}, \bar{x})=$ $\left(\nabla_{p} F(\bar{p}, \bar{x}), \nabla_{x} F(\bar{p}, \bar{x})\right)$, then for any $y^{*} \in K_{u p}^{*}$,

$$
\begin{aligned}
& \hat{D}^{*} \mathcal{F}(\bar{p}, \bar{y})\left(y^{*}\right) \subset\left\{\nabla_{p} F(\bar{p}, \bar{x})^{*} y^{*}\right. \\
+ & \left.p^{*} \mid\left(p^{*},-\nabla_{x} F(\bar{p}, \bar{x})^{*} y^{*}\right) \in \nabla h(\bar{p}, \bar{x})^{*} \hat{N}((\bar{p}, \bar{x}), \Theta)\right\} .
\end{aligned}
$$

(iv) If $F$ is strictly differentiable at $(\bar{p}, \bar{x})$ and $S$ is inner semicontinuous at $(\bar{p}, \bar{y}, \bar{x})$, then for any $y^{*} \in K_{u p}^{*}$,

$$
\begin{aligned}
& D^{*} \mathcal{F}(\bar{p}, \bar{y})\left(y^{*}\right) \\
\subset & \left\{\nabla_{p} F(\bar{p}, \bar{x})^{*} y^{*}+p^{*} \mid\left(p^{*},-\nabla_{x} F(\bar{p}, \bar{x})^{*} y^{*}\right) \in \nabla h(\bar{p}, \bar{x})^{*} N((\bar{p}, \bar{x}), \Theta)\right\} .
\end{aligned}
$$

Moreover, if $S$ admits a local upper Lipschitzian selection at $(\bar{p}, \bar{y}, \bar{x})$, then the converse inclusion of (37) holds. Further, if $F$ is $N$-regular at $(\bar{p}, \bar{x}, \bar{y})$ and $\Theta$ is regular at $(\bar{p}, \bar{x})$, then the converse inclusions of (36) and (38) hold. 
Proof. Observe that the graph of the constraint mapping $C$ in (34) admits the inverse image representation

$$
\operatorname{gph} C=h^{-1}(\Theta):=\{(p, x) \in P \times X \mid h(p, x) \in \Theta\} .
$$

By the strict differentiability of $h$ and the surjectivity assumption on the derivative $\nabla h(\bar{x}, \bar{z})$, we get from Corollary 1.15 in [19] and (39) that

$$
\hat{N}((\bar{p}, \bar{x}) ; \operatorname{gph} C)=\hat{N}\left((\bar{p}, \bar{x}) ; h^{-1}(\Theta)\right)=\nabla h(\bar{p}, \bar{x})^{*} \hat{N}((\bar{p}, \bar{x}), \Theta) .
$$

Then it follows from the definition of Fréchet coderivative that

$$
\hat{D}^{*} C(\bar{p}, \bar{x})\left(x^{*}\right)=\left\{p^{*} \in P^{*} \mid\left(p^{*},-x^{*}\right) \in \nabla h(\bar{p}, \bar{x})^{*} \hat{N}((\bar{p}, \bar{x}), \Theta)\right\} .
$$

Similarly, Theorem 1.17 in [19] ensures that

$$
D^{*} C(\bar{p}, \bar{x})\left(x^{*}\right)=\left\{p^{*} \in P^{*} \mid\left(p^{*},-x^{*}\right) \in \nabla h(\bar{p}, \bar{x})^{*} N((\bar{p}, \bar{x}), \Theta)\right\} .
$$

It further follows from Theorem 1.19 in [19] that the N-regularity of the above mapping $C$ at $(\bar{p}, \bar{x})$ is equivalent, by the inverse image representation and the surjectivity assumption on $\nabla h(\bar{p}, \bar{x})$, to the normal regularity of the set $\Theta$ at $(\bar{p}, \bar{x})$. Therefore, the results immediately follow from Corollary 3.3. This completes the proof.

\section{REFERENCES}

1. T. Q. Bao and B. S. Mordukhovich, Existence of minimizers and necessary conditions in set-valued optimization with equilibrium constraints, Appl. Math., 52 (2007), 453472.

2. E. M. Bednarczuk and W. Song, Contingent epiderivative and its applications to setvalued maps, Control Cybernet, 27 (1998), 375-386.

3. G. Y. Chen and J. Jahn, Optimality conditions for set-valued optimization problems. Set-valued optimization, Math. Meth. Oper. Res., 48 (1998), 187-200.

4. T. D. Chuong and J. C. Yao, Coderivatives of efficient point multifunctions in parametric vector optimization, Taiwanese J. Math., 13(6) (2009), 1671-1693.

5. J. Dutta and C. Tammer, Lagrangian conditions for vector optimization in Banach spaces, Math. Meth. Oper. Res., 64 (2006), 521-541.

6. R. Henrion, Characterization of stability for cone increasing constraints in stochastic programming, Set-Valued Anal., 5 (1997), 323-349.

7. N. Q. Huy, B. S. Mordukhovich and J. C. Yao, Coderivatives of frontier and solution maps in parametric multiobjective optimization, Taiwanese J. Math., 12(8) (2008), 2083-2111. 
8. A. Y. Kruger and B. S. Mordukhovich, Extremal points and the Euler equation in nonsmooth optimization, Dokl. Akad. Nauk BSSR, 24 (1980), 684-687.

9. H. Kuk, T. Tanino and M. Tanaka, Sensitivity analysis in vector optimization, $J$. Optim. Theory Appl., 89 (1996), 713-730.

10. G. M. Lee and N. Q. Huy, On sensitivity analysis in vector optimization, Taiwanese J. Math., 11 (2007), 945-958.

11. A. B. Levy and B. S. Mordukhovich, Coderivatives in parametric optimization, Math. Prog., Ser. A, 99 (2004), 311-327.

12. S. J. Li, H. Yan and G. Y. Chen, Differential and sensitivity properties of gap functions for vector variational inequalities, Math. Meth. Oper. Res., 57 (2003), 377-391.

13. M. H. Li and S. J. Li, Second-order differential and sensitivity properties of weak vector variational inequalities, J. Optim. Theory Appl., 144 (2010), 76-87.

14. Y. Lucet and J. J. Ye, Sensitivity analysis of the value function for optimization problems with variational inequality constraints, SIAM J. Control Optim., 40 (2001), 699-723; Erratum SIAM J. Control Optim., 41 (2002), 1315-1319.

15. K. W. Meng and S. J. Li, Differential and sensitivity properties of gap functions for Minty vector variational inequalities, J. Math. Anal. Appl., 337 (2008), 386-398.

16. B. S. Mordukhovich, Maximum principle in problems of time optimal control with nonsmooth constraints, J. Appl. Math. Mech., 40 (1976), 960-969.

17. B. S. Mordukhovich, Metric approximations and necessary optimality conditions for general classes of extremal problems, Soviet Math. Dokl., 22 (1980), 526-530.

18. B. S. Mordukhovich, Coderivatives of set-valued mappings: calculus and applications, Nonlinear Anal., 30 (1997), 3059-3070.

19. B. S. Mordukhovich, Variational Analysis and Generalized Differentiation, Vol. I: Basic Theory, Vol. II: Applications, Springer-Verlag, Berlin, 2006.

20. B. S. Mordukhovich, N. M. Nam and N. D. Yen, Fréchet subdifferential calculus and optimality conditions in nondifferentiable programming, Optimization, 55 (2006), 685-708.

21. B. S. Mordukhovich, N. M. Nam and N. D. Yen, Subgradients of marginal functions in parametric mathematical programming, Math. Prog., 116 (2009), 369-396.

22. B. S. Mordukhovich, Multiobjective optimization problems with equilibrium constraints, Math. Prog., Ser. B, 117 (2009), 331-354.

23. J. V. Outrata, Optimality conditions for a class of mathematical programs with equilibrium constraints, Math. Oper. Res., 24 (1999), 627-644.

24. R. R. Phelps, Convex Functions, Monotone Operators and Differentiability, 2nd ed., Springer, Berlin, 1993.

25. R. T. Rockafellar, Proto-differentiability of set-valued mappings and its applications in optimization, Annales de l'Institut Henri Poincaré-Analyse non linéaire, 6 (1989), 449-482. 
26. D. S. Shi, Contingent derivative of the perturbation map in multiobjective optimization, J. Optim. Theory Appl., 70 (1991), 385-396.

27. T. Tanino, Stability and sensitivity analysis in convex vector optimization, SIAM J. Control Optim., 26 (1988), 521-536.

28. J. J. Ye and Q. J. Zhu, Multiobjective optimization problem with variational inequality constraints, Math. Prog., Ser. A, 96 (2003), 139-160.

29. R. Zhang, Weakly upper Lipschitzian multifunctions and applications to parametric optimization, Math. Prog., Ser. A, 102 (2005), 153-166.

X. W. Xue, S. J. Li and C. M. Liao

College of Mathematics and Statistics

Chongqing University

Chongqing 401331

P. R. China

E-mail: xuexw1@126.com

lisj@cqu.edu.cn

liaocm520@163.com

Jen-Chih Yao

Center for General Education

Kaohsiung Medical University

Kaohsiung 807, Taiwan

E-mail: yaojc@kmu.edu.tw 